\title{
On the Conversational Basis of Some Presuppositions
}

\author{
Mandy Simons \\ Carnegie Mellon University
}

\section{Introduction}

The current literature on presupposition focuses almost exclusively on the projection problem: the question of how and why the presuppositions of atomic clauses are projected to complex sentences which embed them. Very little attention has been paid to the question of how and why these presuppositions arise at all. As Kay (1992, p.335) observes, "treatments of the presupposition inheritance problem almost never deal with the reasons that individual words and constructions give rise, in the first place, to the particular presuppositions that they do." "This is the question on which this paper will focus.

There are two kinds of answer that one might give to the question of how presuppositions arise. One type of answer is that presuppositions are conventional properties of lexical items, as in the conventional implicature view of Karttunen and Peters (1979). On this view, certain lexical items have, in addition to their truth conditional content, a special presuppositional content, which is carried through the compositional process to produce a propositional presupposition. Although the Karttunen and Peters model for treating presupposition has been rejected by most current researchers, our talk about presupposition seems at least implicitly to take their view of the sources of presuppositions for granted: we talk about the presuppositions of know, of too, and so on, as if assuming that the presuppositions are properties of these items. ${ }^{2}$

Presuppositions, however, might be thought to have a very different source. Presuppositions might be conversationally derived, that is, they might be inferences which are licensed by general conversational principles, in combination with the truth conditions of the presupposing utterance. Stalnaker, from whom we have inherited the currently standard view of presupposition, suggests repeatedly that at least some presuppositions have a conversational source. Indeed, he sees one of the primary advantages of the move from a semantic to a pragmatic account of presupposition as being the possibility of explaining "some of the [presupposition] facts in terms of general assumptions about rational strategy in situations where people exchange information or conduct argument" (1974, p. 205). However, Stalnaker never attempts to work out any general derivation for conversational presuppositions, nor any means for distinguishing conventionally generated presuppositions from those with a conversational source. In the more recent literature, Chierchia and McConnell-Ginet (1990) are the only authors I know of who take seriously the idea of a conversational derivation for presuppositions, but even their treatment is rather brief.

My primary goal in this paper is to motivate the claim that at least some presuppositions have a conversational source, and to set out a partial proposal for the conversational derivation of these presuppositions. Thinking about the derivation of 
presuppositions is worthwhile, I think, because it gives us a somewhat different perspective on what presuppositions are, and raises some questions about standard assumptions.

\section{Evidence for the conversational basis of some presuppositions}

We begin with the evidence that at least some presuppositions do have a conversational source. In this section, I'll show that two classes of presuppositions - the presuppositions of change of state sentences and of factives - possess two properties that are typical of conversational implicature, the paradigm case of a conversationally derived inference. The two properties are contextual defeasibility and nondetachability. We'll take these two in turn.

\subsection{Contextual Defeasibility}

It is well known that all presuppositions can be canceled (fail to project) in certain sentential contexts. In addition, some - but crucially, not all - presuppositions are susceptible to cancellation in what I will call explicit ignorance contexts: situations in which it is apparent to the addressee that the speaker is ignorant with respect to the proposition that would normally be presupposed. This type of case has already been noted in the literature. We begin with an example from Geurts (1994). Imagine a casual conversation taking place between two people who are meeting for the first time. One remarks to the other:

(1) I notice that you keep chewing on your pencil. Have you recently stopped smoking?

In this situation, the addressee knows that the speaker is ignorant of her current or prior smoking habits, and in particular cannot be assuming that she (the addressee) was recently a smoker. In this situation, no implication would arise that the speaker believes the addressee to have been a smoker. The speaker is understood merely as asking whether the addressee has undergone the relevant change of state from being a smoker to not being one, equivalent to:

(2) Is it the case that you have recently been a smoker and have recently ceased to be one?

What is involved here is the choice of a non-presupposing interpretation of the question over a presupposing interpretation. While the presupposing interpretation is usually preferred, we apparently have a stronger preference not to attribute to the speaker an unfounded assumption. So the presuppositional interpretation is not chosen. 
Example (3) is a variation on the same theme. (To make the example natural, assume that there is some special symptom displayed by a person who has stopped smoking that Jane does not display.)

(3) I have no idea whether Jane ever smoked, but she hasn't stopped smoking. ${ }^{3}$

Here, the speaker begins with an explicit assertion of her ignorance about the relevant proposition, and thus the stop clause does not give rise to the presuppositional inference that Jane is or was a smoker. The clause is understood simply as denying that she has undergone the change of state.

Not all expected presuppositions fail to arise in explicit ignorance contexts. In particular, presuppositions which have clearly identifiable lexical triggers such as even, too and again are not cancelable in this way. As an example, suppose I happen to meet a slight acquaintance at a video rental store, someone whose history of video rental I clearly know nothing about. I ask her:

Are you renting "Manhattan" again?

Despite the explicit ignorance context, my addressee would be forced to conclude that I believe for some reason that she has rented "Manhattan" before. In particular, there is no way for her to understand me as asking:

Is it the case the you have rented "Manhattan" before, and are going to do so again?

The presupposition generated by again is not canceled even when the speaker explicitly asserts her ignorance, leading to anomaly in cases like (6):

\# I don't know if Jane ever rented "Manhattan" before, but perhaps she's renting it again.

Contrast (6) with (7), which shows that the anomaly would disappear if a nonpresuppositional reading were available for the second clause.

(7) I don't know if Jane ever rented "Manhattan" before, but perhaps she has and is renting it again.

Examples (1) and (3), which show contextual defeasibility of a presupposition, both involve the presupposition of the change of state verb stop. Indeed, all change of state predicates give rise to presuppositions of the same type, and all these presuppositions are contextually defeasible. ${ }^{4}$ The presuppositions of factives show the same susceptibility to contextual defeasibility, as noted in the literature. Let's set up another explicit ignorance context: Suppose we are at a restaurant, and notice a couple at another table engaged in a furious argument. We are speculating as to what has upset them. I say to you: 
As you know that I couldn't possibly know whether he has in fact been having an affair, you will not take me to presuppose that he has, but merely to be making the relatively weak supposition that he has been having an affair and that she has discovered it.

Chierchia and McConnell-Ginet offer a similar example. They observe that sentence (9) below could well be uttered as part of a conversation between two people who know that Henry is searching for Jane, but who don't themselves know where Jane is:

(9) If Henry discovers that Jane is in New York, there'll be trouble.

In this situation, there is no presupposition or implication that Jane is in fact in NY.

The point of all of these examples is that the presuppositions of change of state predicates and of factives display the same kind of contextual defeasibility as do (generalized) conversational implicatures. The implicatures generated by the Maxim of Quantity are extremely robust, but they too are defeated in explicit ignorance contexts. The examples are familiar. Ordinarily, if I say George has three children, you will understand me to mean that George has exactly three children. However, I can say without contradiction:

(10) George has three children, and may have more for all I know.

The assertion of ignorance in the second conjunct suffices to suppress the usual scalar implicature, just as assertions of ignorance in the presuppositional examples suppressed the expected presuppositions. This, then, is the first piece of evidence that at least some presuppositions have a conversational basis. ${ }^{5}$

\subsection{Nondetachability}

Grice (1967) observes that conversational implicatures are by necessity nondetachable from the content of whatever utterance gives rise to them. What this means is that if utterance of a sentence with a particular content $\mathrm{C}$ generates an implicature in a given conversational context, then utterance of any other sentence with the same content will give rise to the same implicature. ${ }^{6}$ For example, consider the short exchange in (11):

(11) Jane: Do you want to go out for a drink?

Julia: I have to finish writing my SALT paper.

In the context of Jane's question, Julia's utterance generates the implicature that she does not want to go out for a drink. However, the generation of the implicature is not dependent upon the form of Julia's utterance. Any other form which expresses more 
or less the same content would do just as well to produce the implicature. The responses in (12) are all possible candidates.
a. ... I need to finish my SALT paper.
b. ... My SALT paper needs to get finished tonight.
c. ... I have to work on my SALT paper.

Conversational implicatures are nondetachable because they are due to the expression of a particular content in a particular conversational content. They are - by definition - not conventionally associated with any expression, so naturally we do not expect that the expression of the same content in a different form will affect the implicature.

Some presuppositions turn out to have this same property of nondetachability. Consider another example with stop:

Jane didn't stop laughing.

Utterance of this sentence normally gives rise to the presupposition that Jane had been laughing immediately prior to the reference time of the sentence. We standardly say that this is due to the presupposition associated with stop. But notice that if we replace stop with any of its synonyms, the presupposition remains:
a. Jane didn't quit laughing.
b. Jane didn't cease laughing.
c. Jane did not discontinue her laughter.

The same is true of the presuppositions of all other change of state predicates. As further illustration, consider the synonymous sentences in (15). Utterance of any of these would normally give rise to the presupposition that Jane was in the house immediately prior to the reference time of the sentence.
a. Jane didn't leave the house.
b. Jane didn't quit the house.
c. Jane didn't go out of the house.
d. Jane didn't exit the house.
e. Jane didn't depart from the house.

The same point is illustrated with the factive realize and its synonyms in (16).

(16) Harry didn't realize / come to know / become aware that he was a fool.

These observations strongly suggest that the presuppositions of change of state predicates and of factives are nondetachable, that is, that they attach to the content expressed, and not to any lexical item. But presuppositions or implications cannot attach to content by convention. Thus, these presuppositions must have a conversational source. 
Of course, one could deny that the data show nondetachability, and argue instead that in each of these cases, the presupposition that arises is due to a conventional property of one of the lexical items used. However, this immediately raises the question of why all items with the same truth conditional content as, say, stop should also have the same presuppositional content. After all, it could be quite useful for language users to have in the language two items which are truth conditionally equivalent but presuppositionally distinct. In fact, the observation that some set of truth conditionally equivalent forms all give rise to the same presupposition is strong motivation to seek a principled connection between the truth conditional content and the observed presupposition.

\subsection{More on nondetachability}

I must confess that the nondetachability argument is something of a double edged sword for me. The problem is that the very same arguments appear to apply to presuppositions which I would rather assume are in fact conventionally attached to particular lexical items, namely, the presuppositions triggered by even, too, again and the like. For example, all of the sentences in (17) give rise to the presupposition that some (relevant) person other than Harriet is going to SALT.
a. Harriet might go to SALT too.
b. Harriet might go to SALT as well.
c. Harriet might also go to SALT.

The same point can be made about again and its synonyms; the point is harder to make with even, simply because it doesn't have any obvious synonyms in English.

There is, however, a difference between the cases of again and too, and the change of state and factive cases discussed above. It is possible to express the nonpresuppositional content of the sentences in (17) independently of the presupposition, simply by omitting the presupposition trigger. In the case of the change of state predicates and the factives, there is no way to separate these two types of content. On one way of viewing things, this is to say that the presupposition in (17)a-c is not nondetachable: the truth conditional content of these sentences is just that Harriet might go to SALT, and this content can be expressed without giving rise to the presupposition. Hence, the presupposition is not attached to the truth conditional content. The danger of this argument, as Kent Bach has recently pointed out in another context, is that it can become question-begging. It requires the prior assumption that whatever content is contributed by too, as well and also is not part of the truth conditional content of the sentence. However, even if we dismiss this objection and maintain that the presupposition is nondetachable, the question remains why the content expressed by these items is always presuppositional, and never truth conditional.

This problem is, though, tangential to my current purposes. My goal here is to demonstrate that at least some presuppositions have the properties of conversationally generated inferences. The presuppositions of change of state 
predicates and of factives are robustly nondetachable, as well as being contextually defeasible. These observations strongly suggest that at least these presuppositions have a conversational basis.

\section{More evidence: Projection without presuppositions}

I turn now to a second kind of evidence that some general strategy for generating presuppositions is needed. This evidence involves cases where we get something that looks like a presuppositional inference in the absence of anything that we would normally think of as a presupposition trigger. My first set of examples all involve voting for Nader. Let's begin with the following exchange:

Jane: George voted for Nader.

Julia: No he didn't.

Note that Julia's response would normally be taken as a denial of a vote for Nader, not as a denial that George voted at all. The same would generally be true of an utterance of the full negation:

\section{George didn't vote for Nader.}

This implication is easily cancelable, but the cancellation has the "flavor" of presupposition cancellation:

(20) George didn't vote for Nader. In fact, he didn't vote at all.

I must immediately acknowledge that the facts are changed by the introduction of focal stress. Focal stress on George, for example, gives rise to a presupposition or implication that someone other than George voted for Nader, and also seems to reduce the strength of the implication that George voted. Focal stress in other positions similarly changes the presuppositions, in ways that have been well documented in the focus literature. What is crucial for my current purposes is that there is no stress pattern naturally interpreted as a denial that George voted.

In examples (18) and (19), the clause George voted for Nader is embedded under negation. We have observed that one entailment of the clause "projects" over the negation, surviving as a non-entailed implication The same "projection effects" occur in questions, under epistemic modals, and in conditionals, just as with standard presuppositions. Thus, utterance of any of the sentences in (21) would normally give rise to the implication that the speaker believes that George voted:

(21) a. Did George vote for Nader?

b. Perhaps George voted for Nader.

c. If George voted for Nader, he's more politically motivated than he used to be. 
One way to characterize these observations would be to say that the sentence George voted for Nader presupposes that George voted. But to what would we attach this presupposition? It cannot be attached to vote alone, for the "presupposition" disappears when the modifier is removed. Sentence (22) obviously does not presuppose that George voted.

\section{George didn't vote}

On the other hand, there is no mechanism to attach the presupposition to the complex expression vote for Nader, for this expression does not have a lexical entry and is not formed by a construction-specific rule (cf. note 2). Moreover, there is no intuition that the prior assumption that George voted is required in order to make sense of the sentences in (19) and (21). We get little explanatory benefit from saying that George voted for Nader presupposes that George voted. What is wanted is some principled explanation for the inferences observed.

Once we find one example of this kind of projection without presupposition, the cases multiply rapidly. There are two different types of example, which I designate lexical and modificational. Sentences with the verb win are an instance of a lexical case. Consider sentence (23). This entails that Smith will participate in the race:

Smith will win this race.

The sentences in (24) do not share this entailment, but a speaker who uttered either of these sentences would nonetheless normally be taken to believe that Smith will participate in the race. In other words, the entailment that Smith will participate in the race "projects" over the negation.
a. Smith won't win this race.
b. Will Smith win this race?

The adjectives late and early behave in the same way. To say either (25)a. or b. is to imply that Jones has an obligation to be somewhere at a particular time, a proposition which would be entailed by the corresponding affirmative but is not entailed by the negations:
a. Jones isn't late.
b. Jones isn't early.

Many morphologically complex verbs show the same kind of projection effects. ${ }^{7}$ Thus, utterance of any of the following would imply that the soup had been heated:

I didn't reheat / overheat / underheat the soup. 
The same holds for any sentence whose main verb is formed with one of these prefixes.

Admittedly, these are cases where we could in principle attach a conventional presupposition to a lexical form or bound morpheme. Indeed, these cases are reminiscent of examples familiar from the earlier literature on presupposition, which attributed presuppositions to lexical items such as bachelor, spinster and even boy, on the basis of the following patterns of defeasible implication:

(27) Smith isn't a bachelor. Defeasibly implies: Smith is an adult human male. (28) My cousin isn't a boy anymore. Defeasibly implies: My cousin is male.

However, although we could attribute these presuppositional implications to the lexical meaning of the items involved, I find that there is something unsatisfying about saying, for example, that the observed implications of the sentences in (24) are due to a presuppositional specification associated with the verb win. It seems much more plausible that some general principle is involved.

The second class of cases, from which the vote for Nader example is drawn, involve adverbial or adjectival modification. The robustness of the implication in these cases varies, and there may be some disagreement about particular cases. Here are some representative examples:

(29) George isn't arriving on Wednesday.

Defeasibly implies: George is arriving some time.

(30) George didn't leave late.

Defeasibly implies: George left.

(31) I didn't wash the windows with soap.

Defeasibly implies: I washed the windows.

(32) The baby didn't cry loudly.

Defeasibly implies: The baby cried.

The pattern that is emerging here is the following: Given utterance of a sentence of the form $\mathrm{O}[\mathrm{p}]$, where $\mathrm{O}$ is an entailment-canceling operator and where $\mathrm{p}$ entails $\mathrm{q}_{1} \ldots \mathrm{q}_{\mathrm{n}}$, there is a tendency for the utterance to be interpreted as if some subset of $\mathrm{q}_{1} \ldots \mathrm{q}_{\mathrm{n}}$ were outside the scope of $\mathrm{O}^{8}$

I frame this generalization somewhat tentatively. The "projection" interpretations are often preferred, but are not required: hence, the reference to a tendency towards this interpretation. Moreover, not all entailments "project." Among the non-projecting entailments are those which can be identified by replacing the content of an argument position with a variable and existentially closing the result. Thus, an utterance of (33)a. would not normally be interpreted as in (33)b. or c., nor (34)a. as in (34)b. ${ }^{9}$
a. I didn't wash the windows.
b. I washed something, not the windows.
c. Someone washed the windows, not me. 

a. I didn't sleep.
b. Someone slept, but not me.

I do not yet know how projecting entailments should be correctly distinguished from non-projecting ones, and indeed there is a great deal about these cases that I do not yet understand. However, the bottom line is this: In a variety of cases, we find projection behavior where there is no obvious candidate to which to attach a presupposition. And this provides further evidence that some general, conversational principle is responsible for producing projection behavior. ${ }^{10}$

\section{En route to a general principle}

\subsection{An interpretation principle based on logical ordering}

I have now given several arguments that a general principle for generating presuppositional inferences is needed. But what kind of conversational principle would do the job? I cannot yet give a complete answer to this question, but I will here suggest a route towards one. This is a route which I began to pursue only to discover that Stalnaker had already been there before me, albeit just a short way. In speculating as to possible conversational sources of presuppositional constraints, Stalnaker (1974, p.205) suggests that:

The propositions that $\mathrm{P}$ and that $\mathrm{Q}$ may be related to each other, and to common beliefs and intentions, in such a way that it is hard to think of a reason that anyone would raise the question whether $\mathrm{P}$, or care about its answer, unless he already believed that $\mathrm{Q} .{ }^{11}$

The idea is very intuitive. If I say, Jane didn't stop smoking I am showing an interest in the question of whether or not Jane stopped smoking; and why should I care about that question unless I already think that Jane was a smoker? Similarly, I would normally have no reason to wonder who George voted for unless I was already reasonably sure that he voted. The question is whether we can turn this seed of an idea into a robust explanation for the full variety of cases.

There are two issues that must be addressed in order to spell out Stalnaker's idea. The first of these is the issue of when an utterance counts as raising a particular question or as showing interest in the answer to it. Obviously, explicitly asking a question counts as raising it. But offering an answer to a question is also a way to raise it, as suggested above. To say either Jane stopped smoking or Jane didn't stop smoking is to offer an answer to the question Did Jane stop smoking? To offer an answer to a question is to indicate acceptance of that question as a topic of interest, and thereby, in some sense, to raise that question. ${ }^{12}$ Note that because a sentence and its negation both serve to raise the same question, it is expected that they would share any presuppositions generated by virtue of question-raising. It is also expected that 
an explicit utterance of the associated question would give rise to the very same presuppositions. This observation points towards a solution to the projection problem.

The other operators over which presuppositions project are epistemic modals and conditionals. We must therefore ask whether sentences in which a proposition $P$ is embedded under either of these operators can serve to raise the question whether P. It seems that they can. Consider a modal sentence such as:

\section{Perhaps Jane stopped smoking.}

Utterance of this sentence indicates a willingness to discuss the question of whether or not Jane stopped smoking, and certainly indicates an interest in the answer to this question. The modal utterance could indeed be seen as opening an inquiry into the question.

Concerning the antecedents of conditionals, Groenendijk and Stokhof (1984) observe that a conditional can be used to raise the question which would be answered by an assertion of the antecedent. Thus, suppose that I ask whether Jane is likely to get sick, and you reply:

If she doesn't stop smoking, she will get lung cancer.

Although (36) does not provide a direct answer to my question, it suggests a strategy for getting closer to an answer, namely, attempting to answer the question of whether Jane will stop smoking. The conditional thus serves to raise this question. Conditionals also raise the question of the conditions under which the consequent will hold. Thus, if I say:

\section{If Jane isn't wamed of the dangers, she won't stop smoking}

I appear to have an interest in whether or not Jane will stop smoking. The utterance could naturally be followed up with a discussion of what would happen if Jane didn't stop smoking.

It is perhaps unnecessary to establish that each of these individual cases can be seen as raising a question. Searle (1969, p.124) suggests that predication itself involves question-raising: to predicate a property $\mathrm{P}$ of an object $\mathrm{O}$ is to raise the question of whether or not $\mathrm{P}$ is true of o. Searle argues that this is true whatever sentence type the predication is part of, and whatever the speech act function of the utterance as a whole. He says, "the man who asserts that Socrates is wise, the man who asks whether he is wise and the man who requests him to be wise may be said to raise the question of his being wise (of whether "wise" is - or in the case of requests will be - true of him)." On this view, then, all of the cases discussed involve raising the question of whether or not Jane will stop smoking.

The conclusion so far is that it is plausible to think of utterances embedding a proposition $\mathrm{P}$ as raising the question of whether $\mathrm{P}$ is true. This addresses the first issue raised by Stalnaker's suggestion. We turn now to the second, and more 
difficult, issue. Recall that the idea we are pursuing is that two propositions $\mathrm{P}$ and $\mathrm{Q}$ might be related in such a way that it would make sense to raise the question whether $\mathrm{P}$ only if one already believed that $\mathrm{Q}$ was true. The question is this: Just what relation between propositions would impose such a constraint?

As a first stab, it seems likely that the relevant relation is (non-mutual) entailment. After all, in all of the cases that we have considered, the proposition that displays projection behavior is an entailment of the embedded clause. The significance of entailment seems straightforward: if $\mathrm{P}$ entails $\mathrm{Q}$, then $\mathrm{Q}$ is necessary for the truth of $\mathrm{P}$. So it would make sense to establish the truth of $\mathrm{Q}$ before wondering about $\mathrm{P}$. This suggests the following interpretation principle:

\section{Interpretation Principle (tentative)}

Suppose that $\mathrm{P}$ entails but is not entailed by $\mathrm{Q}$. A speaker who raises the question whether $\mathrm{P}$ indicates a belief that $\mathrm{Q}$ is true.

This principle is going to be too strong, but it gets some nice initial results. Let's look at these nice results, and then turn to the problems.

\subsection{Applications}

Let's begin with example (38):

Jane doesn't know that George dislikes Cleo.

This sentence offers a direct answer to the question Does Jane know that George dislikes Cleo?, and so can be said to raise this question. The questioned proposition is the proposition that Jane knows that George dislikes Cleo. Some of the entailments of this proposition are listed in (39).
a. George dislikes Cleo.
b. George exists.
c. Jane exists.
d. Cleo exists.
e. Jane is sentient.
f. George is sentient.

All of these propositions escape the negation in (38), that is, utterance of (38) appears to presuppose these propositions.

There are some less desirable consequences. In addition to the propositions just considered, sentence (38) entails all tautologies, as does every other sentence. Hence, it follows from the Interpretation Principle that utterance of any sentence will give rise to the implication that the speaker believes all tautologies. I will set this point aside as a technicality. In fact, other treatments of presupposition are dogged by the problem of vacuous presuppositions. For example, context change treatments

of presupposition based on Heim (1983) define the presuppositions of a sentence $S$ 
as those propositions entailed by all contexts which admit S. But every consistent context entails all tautologies, and hence on this account too, every sentence presupposes all tautologies.

Let's work through one more well-behaved example:

Jane didn't vote for Nader.

Here, the question raised is:

Did Jane vote for Nader?

The questioned proposition is:

Jane voted for Nader.

This proposition has the entailments listed in (43):
a. Jane exists.
b. Nader exists.
c. Jane voted.
d. Nader was on the ballot. ${ }^{13}$

Again, all of these survive the negation in (40), and emerge as presuppositional implications of its utterance.

\subsection{Problems}

As noted at the outset, the Interpretation Principle is too strong, predicting presuppositional implications which do not occur. We have already seen that $\mathrm{V}+$ complement structures do not show projection behavior, but the Interpretation Principle predicts that they will. Thus, for example, an utterance of the sentence Jane didn't eat the sandwich is incorrectly predicted to imply that the speaker believes that Jane ate something. These entailments, for a reason I do not yet understand, have a different status from those which arise by virtue of adverbial modification.

A different type of problem arises with the change of state verbs on which I have focused attention here. ${ }^{14}$ Consider example (44):

Jane didn't stop laughing.

Sentence (44) raises the question in (45)a., in which the proposition in (45)b. is questioned.

(45) a. Did Jane stop laughing?

b. Jane stopped laughing. 
According to the Interpretation Principle, a speaker who utters (44) should imply that she believes the propositions which are entailed by, but do not entail, (45)b. But these entailments include both of the propositions in (46).

(46) a. Jane was laughing immediately prior to the reference time.

b. Jane was not laughing immediately after the reference time.

Now, the speaker could not coherently assume both of these propositions, given her assertion: she must be taken to assume at most one of these, and to be denying the other. The question is, on what basis does the hearer determine which is which? And in particular, why is it that in almost every case the speaker will be understood to assume that Jane was laughing prior to the reference time, and to be denying that the laughter ceased?

One idea which seems promising is that the two entailments in (46) do not have the same status. Specifically, (46)a. is a precondition of the change of state described by stop laughing. The notion of precondition I intend to invoke here is an ontological one. I conceive of preconditions as holding of events: a precondition on an event $E$ is a condition which must be satisfied by the world in order for the event E to take place. Any event of change of state will have as a precondition that the changing entity be in the appropriate start state for that change of state. ${ }^{15}$ The observation that (46)a. is the usual presupposition of (44) suggests that raising the question of whether a particular change of state took place (or will take place) gives rise to the implication that the speaker believes the relevant start held (or will hold).

While this idea is suggestive, it raises further questions. Why should the precondition have a privileged status relative to other entailments? Can the notion of an ontological precondition be defined for event types other than change of state? Is it the case that all propositions which show projection behavior are characterizable as preconditions? These questions currently remain unanswered.

\subsection{Section Summary}

In this section, I have tried to cash out an idea of Stalnaker's to account for the "projection" of entailments over entailment-canceling operators in a range of cases. The idea, as I have interpreted it, is that an utterance embedding a proposition $\mathrm{P}$ may be understood as raising the question whether P; and P may be related to a further proposition $Q$ in such a way that it would make sense to raise the question whether $\mathrm{P}$ only if $\mathrm{Q}$ were already believed to be true. Hence, utterance of the embedding sentence would give rise to the implication that the speaker believes $\mathrm{Q}$.

In Section 3.1., I argued that the relevant range of utterance types indeed can be seen as raising questions. In Section 3.2., I suggested that the relevant relation between propositions is simply entailment. The discussion in Section 3.3. makes clear that this suggestion needs further refinement. However, the idea that the projection facts may be amenable to a treatment in terms of logical ordering remains attractive. 


\section{Conclusion}

What we have established in this discussion is that at least some presuppositions must have a conversational basis, that is, they must be inferences derivable from some general conversational principle or principles. I would like to conclude by considering what consequences this conclusion has for the general treatment of presupposition.

On the view of presupposition now most standard in the literature, presuppositions are thought to be propositions which must be entailed by the presumed common ground of the discourse participants. However, if at least some presuppositions are derived by the kind of mechanism which gives rise to other conversational inferences (i.e. conversational implicatures), then it is more appropriate to view them as propositions which the addressee can infer the speaker to believe on the basis of what the speaker has said, plus the assumption that the speaker is behaving cooperatively. ${ }^{16}$ On this picture, the derivation of a presupposition may require speaker and addressee to share certain assumptions (e.g. that the speaker is behaving cooperatively), but the presuppositions themselves are neither required nor expected to be entailed by the common ground.

Moreover, on this picture, presuppositions are not attached to atomic clauses, but are inferences derivable from the utterance as a whole, given the conversational situation. This raises a question about algorithmic treatments of presupposition projection, which are predicated on the assumption that presuppositions are locally generated.

This discussion also suggests the possibility that presuppositional phenomena are not homogeneous. If the hypothesis presented here is correct, then the presuppositions of, say, change of state sentences are derived very differently from the presuppositions generated by even, too, and again, which plausibly have a conventional source. On the other hand, this picture of (some) presuppositions as conversationally generated inferences suggests the possibility of unifying the treatment of these presuppositional data with that of other types of "preferred readings," such as those illustrated in Section 2.

Current treatments of presupposition in the dynamic semantics literature have a great deal of appeal. They are formally rigorous, make clear predictions, and are very successful with respect to the range of data which they cover. Unfortunately, these accounts disassociate the question of presupposition projection from the prior question of the sources of presuppositions. The investigation of this question leads us down the notoriously swampy path of conversational inference and interpretative strategies. However, I think that we will not really understand presupposition until we see where this path leads.

\section{Endnotes}

* I have presented some of the ideas discussed in this paper at colloquia at the University of Pennsylvania and at Rutgers University. I am grateful to the audiences 
at those events for comments and discussion, from which this project has benefitted My thanks also to Sally McConnell-Ginet for helping to get this project off the ground. None of the above bears any responsibility for what is amiss in the content of this paper.

1. Kay adds with regret that his own study "will unfortunately make no improvement on this practice."

2. We also attribute presuppositions to specific constructions, in particular it-clefts and wh-clefts. But it is particularly problematic to think of these presuppositions as conventional, given the assumption that there is neither a "lexicon" of constructions, nor any construction-specific syntactic rules. If there is no rule for the formation of clefts, then there is nothing to which to attach the conventional rule that a cleft has a presupposition. (This observation is due to Sally McConnell-Ginet.)

3. Notice that this sentence does not have the usual form of a presuppositioncanceling conjunction. Cancellation is expected when the first conjunct entails the presupposition of the second, which is not the case here. This type of sentence is, however, reminiscent of examples with but due to Liberman (1973), such as:

(i) Perhaps John has no children, but perhaps his children are away on vacation.

Liberman offers his examples as counterexamples to the standard characterization of projection patterns. I think in fact that these examples involve cancellation in the face of explicit ignorance. The first clause indicates that the speaker is not sure whether John has children, and thus has the same effect as the first clause in my example (3).

4. For a more detailed discussion of the presuppositions of change of state sentences, see my manuscript, "Why Some Presuppositions are Conversational Implicatures".

5. Chierchia and McConnell-Ginet (1990) also take contextual defeasibility to be a distinguishing feature of conversationally generated presuppositions.

6. An exception must be made here for Manner implicatures.

7. This observation is due to Tom Werner.

8. In addition, whatever is "scoped out" tends to be interpreted as "backgrounded," as is usual with presupposition. I will have nothing further to say about this here.

9. These readings become available with the right placement of focal stress.

10. Restrictive relatives are another source of projecting entailments, as has been pointed out in the literature. (See Wilson and Sperber 1979 and Chierchia and McConnell-Ginet 1990.) Utterance of either (i) or (ii) commits the speaker to the claim that Jane was hiding in the closet.

(i) I didn't see Jane, who was hiding in the closet.

(ii) Did you see Jane, who was hiding in the closet?

Similarly, in sentences with secondary predication, the primary predication projects. Thus, utterance of either (iii) or (iv) implies that the speaker believes that Cleo came home.

(iii) Cleo didn't come home drunk.

(iv) Did Cleo come home drunk? 
11. It is worth noting that Stalnaker himself here invokes speaker beliefs, not speaker presuppositions, as a condition on appropriate utterance.

12. This is an idea which originates in the work of L. Carlson (1983), who suggested that sentence topics be treated as questions which are answered by utterances of that sentence. The idea has since been pursued by a variety of researchers. See, among others, van Kuppevelt (1995), Ginzburg (1995, 1997), and Roberts (1996) and also Simons (2000).

13. This is not a logical entailment, but a contextual entailment, in the sense of Chierchia and McConnell-Ginet (1990). It follows from (42) given what we know about the world and about how voting works

14. This problem was pointed out to me by Roger Schwarzschild.

15. This notion of preconditions on events is to be distinguished from preconditions on the truth and falsity of sentences. I claim, for example, that Jane's laughing immediately before $t$ is a precondition on the event of her stopping laughing. However, this claim does not entail any commitments as to whether the sentence Jane didn't stop laughing (at $\mathrm{t}$ ) is true, false, or truth valueless in a situation in which the precondition is not met.

16. An alternative view, which I hope to explore further, is suggested by Levinson's recent treatment (Levinson 2000) of Generalized Conversational Implicature. Levinson argues that GCIs are default interpretations produced by interpretative strategies which hearers tend to adopt. According to Levinson, GCIs are distinguished from particularized conversational implicatures in that their calculation does not involve the hearer reasoning about the speaker's beliefs. Presuppositional inferences share the robustness of GCIs, and it is plausible to think that they are generated in the same fashion.

\section{References}

Bach, K. (1999). "The Myth of Conventional Implicature." Linguistics and Philosophy Vol.22 No.4: 327-366.

Carlson, L. (1983). Dialogue Games: An Approach to Discourse Analysis. Boston:

D. Reidel Publishing Company.

Chierchia, G. and S. McConnell-Ginet (1990). Meaning and Grammar, Mass.: MIT Press.

Gazdar, Gerald (1979). Pragmatics: Implicature, Presupposition and Logical Form. New York: Academic Press.

Geurts, B. (1994). Presupposing, $\mathrm{PhD}$ dissertation, Universität Osnabrück.

Ginzburg, Jonathan (1997). "Querying and Assertion in Dialogue". Ms, Hebrew University of Jerusalem.

Grice, Paul (1967). Logic and Conversation. Harvard: the William James Lectures. [Published in Grice (1989)].

Grice, P. (1989). Studies in the Way of Words, Cambridge, Mass.: Harvard University Press. 
Groenendijk, J. and M. Stokhof(1984). Studies on the Semantics of Questions and the Pragmatics of Answers, PhD Dissertation, University of Amsterdam.

Karttunen, L. and S. Peters (1979). "Conventional Implicature." In Oh, C.-K. and D. A. Dinneen, (eds) Syntax and Semantics Vol. 11: Presupposition, NY: Academic Press.

Kay, P. (1992). “The Inheritance of Presuppositions," Linguistics and Philosophy 15: 333-379.

Kuppevelt, J. van (1995). "Discourse Structure, Topicality and Questioning." Journal of Linguistics 31: 109-147.

Levinson, S. (2000). Presumptive Meanings. Cambridge, MA.: MIT Press.

Liberman, M. (1973). "Alternatives." CLS 9: 346-355.

Roberts, C. (1996). "Information Structure in Discourse: Towards an Integrated Formal Theory of Pragmatics." OSU Working Papers in Linguistics 49: 91136.

Searle, J.R. (1969). Speech Acts. NY: Cambridge University Press.

Simons, M. (2000). Issues in the Semantics and Pragmatics of Disjunction. NY: Garland.

Simons, M. (ms). "Why Some Presuppositions are Conversational Implicatures."

Stalnaker, R. (1974). "Pragmatic Presuppositions," In Milton K. Munitz and Peter K. Unger (eds.), Semantics and Philosophy. New York: New York University Press.

Wilson, D. and D. Sperber (1979). "Ordered Entailments: An Alternative to Presupposition Theories." In Oh, C.-K. and D. A. Dinneen, (eds) Syntax and Semantics Vol. 11: Presupposition, NY: Academic Press. 